\title{
Modeling Hidden Exposures in Claim Severity via the EM Algorithm
}

Grzegorz A. Rempala and Richard A. Derrig 


\title{
Modeling Hidden Exposures in Claim Severity via the EM Algorithm
}

\author{
Grzegorz A. Rempala \\ Department of Mathematics, University of Louisville, \\ Richard A. Derrig \\ Automobile Insurers Bureau of Massachusetts
}

\begin{abstract}
We consider the issue of modeling the so-called hidden severity exposure occurring through either incomplete data or an unobserved underlying risk factor. We use the celebrated EM algorithm as a convenient tool in detecting latent (unobserved) risks in finite mixture models of claim severity and in problems where data imputation is needed. We provide examples of applicability of the methodology based on real-life auto injury claim data and compare, when possible, the accuracy of our methods with that of standard techniques.
\end{abstract}

\section{Introduction}

Actuarial analysis can be viewed as the process of studying profitability and solvency of an insurance firm under a realistic and integrated model of key input random variables such as loss frequency and severity, expenses, reinsurance, interest and inflation rates, and asset defaults. In a modern analysis of financial models of property-casualty companies, these input variables typically can be classified into financial market variables and underwriting variables (cf. e.g., D'Arcy et al. 1997). The financial variables generally refer to asset-side generated cash flows of the business, and the underwriting variables relate to the cash flows of the liabilities side. The process of developing any actuarial model begins with the creation of probability distributions of these input variables, including the establishment of the proper range of values of input parameters. The use of parameters is generally determined by the use of the parametric families of distributions, although the non-parametric techniques have a role to play as well (see, e.g., Derrig, et al. 2001). In this article we consider an issue of hidden or "lurking" risk factors or parameters and point out the possible use of the celebrated EM algorithm to uncover those factors. We begin by addressing the most basic questions concerning hidden loss distributions. To keep things in focus we will be concerned here only with two applications to modeling the severity of loss, but the methods discussed may be easily applied to other problems like loss frequencies, asset returns, asset defaults, and combining those into models of Risk Based Capital, Value at Risk, and general Dynamic 
Financial Analysis, including Cash Flow Testing and Asset Adequacy Analysis. Our applications will illustrate the use of the EM algorithm (i) to impute missing values in an asset portfolio and (ii) to screen medical bills for possible fraud or abusive practices.

\subsection{Hidden Exposures in Loss Severity Distributions}

In many instances one would be interested in modeling hidden risk exposures as additional dimen$\operatorname{sion}(\mathrm{s})$ of the loss severity distribution. This in turn in many cases leads to considering mixtures of probability distributions as the model of choice for losses affected by hidden exposures; some parameters of the mixtures will be considered missing (i.e., unobservable in practice). During the last 20 years or so there has been a considerable advancement in statistical methodologies dealing with partially hidden or incomplete data models. Empirical data imputation has become more sophisticated and the availability of ever faster computing power have made it increasingly possible to solve these problems via iterative algorithms.

In our paper we shall illustrate a possible approach to two types of problems arising often in practical situations of modeling the severity of losses: (i) imputation of partially missing multivariate observations and (ii) identification of latent risks via fitting finite mixtures models.

The common feature of both of these issues is, generally speaking, the unavailability of complete information on the variables or parameters of interest. The statistical methodology which is especially well-suited for this type of circumstances is the so-called EM algorithm.

\subsection{The EM Algorithm}

In their seminal paper Dempster, Laird and Rubin (1977) have proposed the methodology which they have called the Expectation-Maximization (EM) algorithm as an iterative way of finding maximum likelihood estimates. ${ }^{1}$ They demonstrated that the method was especially appropriate for finding the parameters of an underlying distribution from a given data set where the data was incomplete or had missing values. At present there are two basic applications of the EM methodology considered in the statistical literature. The first occurs when the data indeed has missing values, due to problems with or limitations of the data collection process. The second occurs when the original likelihood estimation problem is altered by assuming the existence of the hidden parameters or factors. It turns out that both these circumstances can be, at least initially, described in the following statistical setting. Let us consider a density function (possibly multivariate) $p(\cdot \mid \Theta)$ that is indexed by the set of parameters $\Theta$. As a simple example we may take $p$ to be a univariate Gaussian density and $\Theta=\{(\mu, \sigma) \mid-\infty<\mu<\infty, \sigma>0\}$. Additionally, we have an observed data set $\mathcal{X}$ of size $n$, drawn from the distribution $p$. More precisely, we assume that the points of $\mathcal{X}=\left(x_{1}, \ldots, x_{n}\right)$ are the realizations of some independent random variables distributed according to $p(\cdot \mid \Theta)$. We shall call $\mathcal{X}$ the incomplete data. In addition to $\mathcal{X}$, we also

\footnotetext{
${ }^{1}$ A full explanation of the role of the EM algorithm in missing data problems can be found in Little and Rubin, (1987) or in a somewhat more mathematically advanced monograph by McLachlan and Krishnan (1997).
} 
consider a complete data set $\mathcal{Z}=(\mathcal{X}, \mathcal{Y})$ and specify the joint density

$$
p(\mathbf{z} \mid \Theta)=p(\mathbf{x}, \mathbf{y} \mid \Theta)=p(\mathbf{y} \mid \mathbf{x}, \Theta) p(\mathbf{x} \mid \Theta) .
$$

As we can see from the last equality, this joint density $p(\mathbf{z} \mid \Theta)$ arises from considering the marginal density $p(\mathbf{x} \mid \Theta)$ and the specific assumptions on the relation between hidden (or missing) variables $\mathcal{Y}=\left(\mathbf{y}_{1}, \ldots \mathbf{y}_{n}\right)$ and the observed incomplete data $\mathcal{X}$. Associated with the joint density is the joint likelihood function

$$
\mathcal{L}(\Theta \mid \mathcal{Z})=\mathcal{L}(\Theta \mid \mathcal{X}, \mathcal{Y})=\prod_{i=1}^{n} p\left(\mathbf{x}_{i}, \mathbf{y}_{i} \mid \Theta\right)
$$

which is often called the complete likelihood. For the sake of computational simplicity it is often more convenient to consider the logarithm of the complete likelihood

$$
l(\Theta \mid \mathcal{Z})=\log \mathcal{L}(\Theta \mid \mathcal{X}, \mathcal{Y})=\sum_{i=1}^{n} \log p\left(\mathbf{x}_{i}, \mathbf{y}_{i} \mid \Theta\right)
$$

Note that the function above may be thought of as a random variable since it depends on the unknown or missing information $\mathcal{Y}$ which by assumption is governed by an underlying probability distribution. Note also that in accordance with the likelihood principle, we now regard $\mathcal{X}$ as constant.

The EM algorithm as described in Dempster, Laird and Rubin (1977) consists of two steps repeated iteratively. In its expectation step or the E-step, the algorithm first finds the expected value of the complete $\log$-likelihood function $\log p(\mathcal{X}, \mathcal{Y} \mid \Theta)$ with respect to the unknown data $\mathcal{Y}$ given the observed data $\mathcal{X}$ and the current parameter estimates. That is, instead of the complete log-likelihood (2) we consider the following

$$
Q\left(\Theta, \Theta^{(i-1)}\right)=E\left[\log p(\mathcal{X}, \mathcal{Y} \mid \Theta) \mid \mathcal{X}, \Theta^{(i-1)}\right]
$$

Note the presence of the second argument in the function $Q\left(\Theta, \Theta^{(i-1)}\right)$. Here $\Theta^{(i-1)}$ stands for the current value of the parameter $\Theta$ at the iteration $(i-1)$, that is, the value which is used to evaluate the conditional expectation.

After the completion of the E-step, the second step of the algorithm is to maximize the expectation computed in the first step. This is called the maximization or the M-step, at which time the value of $\Theta$ is updated by taking

$$
\Theta^{(i)}=\underset{\Theta}{\operatorname{argmax}} Q\left(\Theta, \Theta^{(i-1)}\right)
$$

The steps are repeated until convergence. It can be shown (via the relation (1) and Jensen's 
inequality) that if $\Theta^{*}$ maximizes $Q\left(\Theta, \Theta^{(i-1)}\right)$ with respect to $\Theta$ for fixed $\Theta^{(i-1)}$ then

$$
l\left(\Theta^{*} \mid \mathcal{Z}\right)-l\left(\Theta^{(i-1)} \mid \mathcal{Z}\right) \geq Q\left(\Theta^{*}, \Theta^{(i-1)}\right)-Q\left(\Theta^{(i-1)}, \Theta^{(i-1)}\right) \geq 0
$$

and each iteration of the procedure indeed increases the value of complete log-likelihood (2). Let us note that from the above argument it follows that a full maximization in the M-step is not necessary: it suffices to find any value of $\Theta^{(i)}$ such that $Q\left(\Theta^{(i)}, \Theta^{(i-1)}\right)>Q\left(\Theta^{(i-1)}, \Theta^{(i-1)}\right)$. Such procedures are called GEM (generalized EM) algorithms. For a complete set of references see, for instance, the monograph by McLachlan and Krishnan (1997) where also the issues of convergence rates for the EM and GEM algorithms are thoroughly discussed. For some additional references and examples see also Wu (1983) or the monographs by Little and Rubin (1987) and Hastie, Tibshirani, and Friedman (2001).

\section{Modeling Hidden Risks via the EM Algorithm}

As indicated in the previous section the primary application of the EM algorithm is in fitting the maximum likelihood models. Since this is accomplished by the M-step of the algorithm, the role of the E-step is, therefore, secondary - it is needed to facilitate the performance of the M-step in the presence of the missing or incomplete data. However, as in this paper we shall focus on the usefulness of the EM procedure in modeling hidden risks or variables, in our setup we shall be in fact more interested in the E-step of the algorithm, as it will provide us with the way to estimate or impute missing data and uncover hidden factors and variables. In our examples below we shall consider two types of hidden (latent) variables. The first one will arise when, due to some problems with the data collection, parts of the observations are missing from the observed dataset. We consider this problem via the EM method in the particular context of multivariate (loss) models.

\subsection{Multivariate Severity Distributions. Data Imputation with EM}

Although insurance has been traditionally build on the assumption of independence and the law of large numbers has governed the determination of premiums, the increasing complexity of insurance and reinsurance products has lead over past decade to increased actuarial interest in the modeling of dependent risks (see, e.g., Wang 1998 or Embrechts et al. 2000). Multivariate loss and risk models (and especially those based on elliptically contoured distributions) have been hence of interest in such areas as Capital Asset Pricing Model and the Arbitrage Pricing Theory (cf. e.g., Campbell, Lo, and Mackinlay 1996).

In some circumstances, however, parts of the observed multivariate data may be missing. Claim reporting systems depend heavily on the front-line adjusters to provide data elements beyond the simple payment amounts. In the absence of, or even in the presence of, system edits, daily work load pressures and the lack of interest in the coded data provide a deadly combination of disincentives for accurate and complete coding. Actuaries are quite familiar with missing data fields, which when 
Table 1: 10 fictitious observed gains and losses from two risk portfolios in thousands.

\begin{tabular}{cccccccccc}
\hline 0.914 & 2.088 & 2.644 & 0.477 & -1.940 & -0.245 & 0.362 & 1.147 & $?$ & $?$ \\
3.855 & 4.025 & 2.092 & 3.400 & 1.520 & 2.626 & $?$ & $?$ & 5.473 & 6.235 \\
\hline
\end{tabular}

essential to the analysis most often results in throwing the record out, thereby creating unknown 'hidden' biases. Likewise, financial time series data may be interrupted, unavailable, or simply lost for securities or portfolios that are not widely tracked.

As an illustration of an application of the EM algorithm in this setting let us consider a hypothetical example of 10 losses/gains from a two-dimensional vector of risk portfolios, which we have generated using a bivariate normal distribution. The data is presented in Table 1 (in thousands of dollars). As we can see parts of the last four observations are missing from the table. In fact, for the purpose of our example, they have been removed from the generated data. We shall illustrate the usefulness of the EM algorithm in estimating these missing values.

If we denote by $\mathcal{X}$ the observed (incomplete) data listed in Table 1 then following our notation from previous section we have the complete data vector $\mathcal{Z}$ given by

$$
\mathcal{Z}=\left(\mathbf{z}_{1} \ldots \mathbf{z}_{n}\right)=\left(\mathbf{x}_{1} \ldots, \mathbf{x}_{6},\left(x_{1,7}, y_{2,7}\right)^{T},\left(x_{1,8}, y_{2,8}\right)^{T},\left(y_{1,9}, x_{2,9}\right)^{T},\left(y_{1,10}, x_{2,10}\right)^{T}\right)
$$

where $\mathrm{x}_{j}=\left(x_{1, j}, x_{2, j}\right)^{T}$ for $j=1 \ldots, 6$ is the set of pairwise complete observations. The missing data (corresponding to ? marks in Table 1 ) is, therefore,

$$
\mathcal{Y}=\left(y_{2,7}, y_{2,8}, y_{1,9}, y_{1,10}\right)
$$

Let us note that under our assumption of normality, the equation (2) now becomes

$$
l(\Theta \mid \mathcal{Z})=-n \log (2 \pi)-\frac{1}{2} \log |\Sigma|-\frac{1}{2} \sum_{j=1}^{n}\left(\mathbf{z}_{j}-\mu\right)^{T} \Sigma^{-1}\left(\mathbf{z}_{j}-\mu\right)
$$

where $n=10, \mu=\left(\mu_{1}, \mu_{2}\right)^{T}$ is a vector of means and

$$
\Sigma=\left(\begin{array}{ll}
\sigma_{11} & \sigma_{12} \\
\sigma_{12} & \sigma_{22}
\end{array}\right)
$$

is covariance matrix. The vector of unknown parameters, therefore, can be represented as

$$
\Theta=\left(\mu_{1}, \mu_{2}, \sigma_{11}, \sigma_{12}, \sigma_{22}\right) \text {. }
$$


In order to describe the EM algorithm in this setting we need to find the particular form of $Q\left(\Theta, \Theta^{(i-1)}\right)$ defined by (3). Due to the independence of the $z_{i}$ 's this is equivalent, in effect, to evaluating

$$
E_{\Theta^{(i-1)}}(Y \mid \mathcal{X}) \quad \text { and } \quad E_{\Theta^{(i-1)}}\left(Y^{2} \mid \mathcal{X}\right)
$$

where $Y$ is the underlying random variable for $\mathcal{Y}$, assumed to be normal. From the general formulae for conditional moments of a bivariate normal variable $X=\left(X_{1}, X_{2}\right)$ with the set of parameters $\Theta$ as above, we have that

$$
\begin{aligned}
E\left(X_{2} \mid X_{1}=x_{1}\right) & =\mu_{2}+\sigma_{12} / \sigma_{11}\left(x_{1}-\mu_{1}\right) \\
\operatorname{Var}\left(X_{2} \mid X_{1}=x_{1}\right) & =\sigma_{22.1}=\sigma_{22}\left(1-\rho^{2}\right)
\end{aligned}
$$

where $\rho$ stands for the correlation coefficient. Interchanging the subscripts 1 and 2 in (7) gives the formulae for the conditional mean and variance of the distribution $X_{1} \mid X_{2}=x_{2}$. Using the relations (7) and the usual formulae for ML estimators of the normal mean vector $\mu$ and the covariance matrix $\Sigma$, we may now state the EM algorithm for imputing missing data in Table 1 as follows.

\section{Algorithm 1 (EM version of Buck's algorithm)}

1. Define the initial value $\Theta^{(0)}$ of the set of parameters (5). Typically, it can be obtained on the basis of the set of complete pairs of observations (i.e., $\mathbf{x}_{1} \ldots, \mathbf{x}_{6}$ in Table 1).

2. The E-step: given the value of $\Theta^{(i)}$ calculate via (7) the vector $\mathcal{Y}^{(i)}$ of the imputations of the missing data $\mathcal{Y}$ given by (6).

$$
\begin{aligned}
& y_{2 k}^{(i)}=\mu_{2}^{(i)}+\frac{\sigma_{12}^{(i)}}{\sigma_{11}^{(i)}}\left(x_{1 k}-\mu_{1}^{(i)}\right) \quad \text { and } \quad y_{2 k}^{2}(i)=\left(y_{2 k}^{(i)}\right)^{2}+\sigma_{22.1}^{(i)} \quad \text { for } k=7,8 \\
& y_{1 k}^{(i)}=\mu_{1}^{(i)}+\frac{\sigma_{12}^{(i)}}{\sigma_{22}^{(i)}}\left(x_{2 k}-\mu_{2}^{(i)}\right) \quad \text { and } \quad y_{1 k}^{2(i)}=\left(y_{1 k}^{(i)}\right)^{2}+\sigma_{11.2}^{(i)} \quad \text { for } k=9,10
\end{aligned}
$$

3. The M-step: given the current value of the imputed complete data vector $\mathcal{Z}^{(i)}=\left(\mathcal{X}, \mathcal{Y}^{(i)}\right)$ set $M_{k}=\sum_{j=1}^{n} z_{k, j}^{(i)} / n$ and $M_{k l}=\sum_{j=1}^{n} z_{k, j}^{(i)} z_{l, j}^{(i)} / n$ for $k, l=1,2$, and calculate $\Theta^{(i+1)}$ as

$$
\begin{aligned}
\left(\mu_{1}^{(i+1)}, \mu_{2}^{(i+1)}\right) & =\left(M_{1}, M_{2}\right) \\
\sigma_{k l}^{(i+1)} & =M_{k l}-M_{k} M_{l} \quad \text { for } k, l=1,2
\end{aligned}
$$

4. Repeat steps 2 and 3 until the relative difference of the subsequent values of $l\left(\Theta^{(i+1)} \mid \mathcal{Z}^{(i)}\right)$ is sufficiently small. 
The above algorithm in its non-iterative version was first introduced by Buck (1960) who used the method of imputation via linear regression with subsequent covariance correction to estimate means and covariance matrices of $p$ dimensional random vectors in case when some parts of the vector components were missing. For more details about Buck's imputation procedure, we refer to his original paper (Buck 1960) or to Chapter 3 of Little and Rubin (1987) or Chapter 2 of MeLachlan and Krishnan (1997).

The numerical illustration of the algorithm is presented in Table 2. As we can see from the

Table 2: Selected iterations of the EM algorithm for data in Table 1.

\begin{tabular}{c|ccccccccc|c}
\hline Iteration & $\mu_{1}$ & $\mu_{2}$ & $\sigma_{11}$ & $\sigma_{12}$ & $\sigma_{22}$ & $y_{2,7}$ & $y_{2,8}$ & $y_{1,9}$ & $y_{1,10}$ & $-2 Q$ \\
\hline 1 & 0.6764 & 3.5068 & 1.8170 & 0.3868 & 2.0671 & 3.4399 & 3.6069 & 1.0443 & 1.1867 & 65.7704 \\
5 & 0.8779 & 3.6433 & 1.8618 & 0.8671 & 2.2030 & 3.4030 & 3.7685 & 1.5982 & 1.8978 & 64.7568 \\
10 & 0.9279 & 3.6327 & 1.9463 & 0.9837 & 2.1724 & 3.3466 & 3.7433 & 1.7614 & 2.1061 & 64.5587 \\
20 & 0.9426 & 3.6293 & 1.9757 & 1.0181 & 2.1639 & 3.3301 & 3.7345 & 1.8102 & 2.1683 & 64.5079 \\
30 & 0.9435 & 3.6291 & 1.9775 & 1.0202 & 2.1634 & 3.3291 & 3.7339 & 1.8132 & 2.1722 & 64.5048 \\
35 & 0.9436 & 3.6291 & 1.9776 & 1.0203 & 2.1633 & 3.3290 & 3.7339 & 1.8134 & 2.1724 & 64.5047 \\
40 & 0.9436 & 3.6291 & 1.9777 & 1.0204 & 2.1633 & 3.3290 & 3.7339 & 1.8134 & 2.1724 & 64.5046 \\
45 & 0.9436 & 3.6291 & 1.9777 & 1.0204 & 2.1633 & 3.3290 & 3.7339 & 1.8134 & 2.1725 & 64.5046 \\
\hline
\end{tabular}

table with the accuracy of up to three significant digits, the algorithm seems to converge after about $\mathbf{3 0}$ steps or so and the estimated or imputed values of (5) are given by

$$
\mathcal{Y}^{(e m)}=(3.329,3.734,1.813,2.173) .
$$

Let us note, for the sake of comparison, that if we were to employ the standard, "naive" linear or polynomial regression model based on 6 complete observations in order to fit the missing values in Table 1 we would have obtained in this case

$$
\mathcal{Y}^{(r e g)}=(2.834,3.063,2.700,3.269) .
$$

Both $\mathcal{Y}^{(e m)}$ and $\mathcal{Y}^{(r e g)}$ can be now compared with the actual values removed from Table 1 which were

$$
\mathcal{Y}=(3.362,3.657,1.484,3.410) \text {. }
$$

As we can see, in our example the EM method did reasonably well in recovering the missing values. 


\subsection{Massachusetts Auto Bodily Injury Liability Data. Fraud and Build-up Screen- ing via Mixture Models}

By now it is fairly well known that fraud and build-up, exaggerated injuries and/or excessive treatment, are key components of the auto injury loss distributions (Derrig et al. 1994, Cummins and Tennyson 1996, Abrahamse and Carroll 1999). Indeed, injury loss distributions are prime candidates for mixture modeling, for at least the differing of payment patterns by injury type. Even within an injury type as predominant as strain and sprain, ${ }^{2}$ there can be substantial differences in subpopulations arising from fraud and build-up. One common method of identifying these claims has been to gather additional features of the claim, the so-called fraud indicators, and to build models to identify those bogus claims (Brockett, et al. 1998). The acquisition of reliable indicators some of which may be highly subjective, is costly, and may not be efficient in uncovering abusive patterns in injury claims (Crocker and Tennyson 1999). The use of more flexible methods such as the fuzzy logic (see more below) may overcome the lack of this precision in subjective features in an economically efficient manner by running a background algorithm on adjusters' electronic files (see, for example, Derrig and Ostaszewski 1995, 1999).

Another approach to uncovering fraud and build up, perhaps grounded more in practical considerations, is to construct a filter, or screening algorithm, for medical provider bills (Derrig 2002). Routinely, excessive medical bills can be reduced to "reasonable and customary" levels by computer algorithms that compare incoming bills to right censored billing distributions with "excessive" being operationally defined to be above the censoring point. Less routine is the implementation of systematic analysis of the patterns of a provider's billing practices (Major and Riedinger 1992). Our second application of the EM algorithm is to build a first level screening device to uncover potential abusive billing practices and the appropriate set of claims to review. We perform the pattern analysis by uncovering abusive-like distributions within mixture models parametrized by the estimates obtained via the EM algorithm. An illustration of the method follows.

In the table provided in Appendix B we present a set of outpatient medical provider's total billings on the set of 348 auto bodily injury liability claims closed in Massachusetts during 2001. For illustration purposes, 76 claims with one "outlier" provider ("A") were chosen based on a pattern typical of abusive practice; namely, an empirical kurtosis more than five times the overall average. The "outlier" was then combined with medical bills in claims from a random sample of providers. The losses are recorded in thousands and are presented in column two. Column 4 identifies each medical billing amount as provider " $A$ " or "other". We will use the EM algorithm applied to a normal $(\mathrm{log})$ mixture model attempting to uncover provider $\mathrm{A}$.

The relatively large volume of provider A's claims is clearly visible in the left panel of Figure 1, where it is presented as a portion of the overall claims

Whereas the volume of claims by itself never constitutes a basis for the suspicion of fraud or build-up, it certainly might warrant a closer look at the data at hand, especially via some type of

\footnotetext{
${ }^{2}$ Currently, Massachusetts insured bodily injury claims are upwards of 80 percent strain and sprain claims as the most costly part of the medical treatment. Of course, that may have a dependency on the $\$ 2,000$ dollar threshold to file a tort claim.
} 
Figure 1: Overall distribution of the $348 \mathrm{BI}$ medical bill amounts from Appendix B compared with that submitted by provider A. Left panel: frequency histograms (provider A's histogram in filled bars). Right panel:density estimators (provider A's density in dashed line).
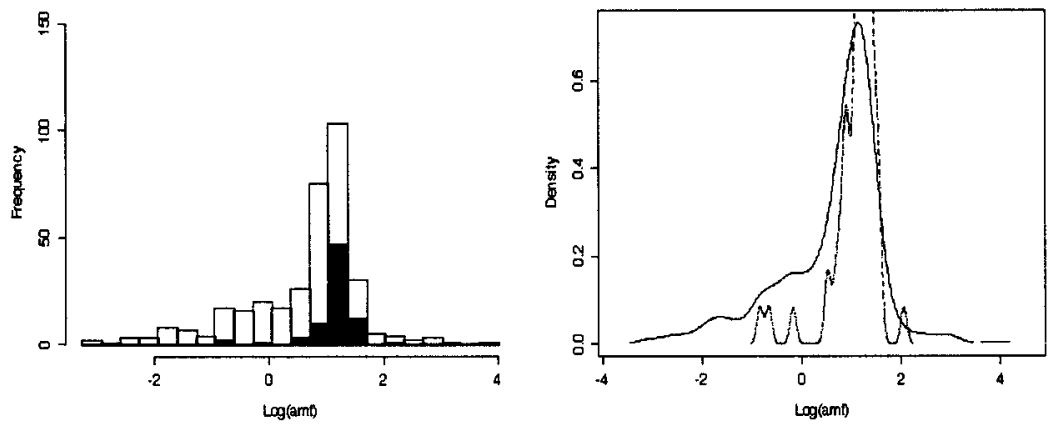

homogeneity analysis, since the second panel in Figure 1 clearly indicates the difference between the overall claims distribution and that of the provider $A$. Hence in this problem we shall be looking for a hidden exposure which could manifest itself as a non-homogenous component of the data, albeit we shall not be assuming that this component is necessarily due to provider $A$. In fact, as the initial inspection of the overall data distribution does not immediately indicate non-homogeneity we shall not make any prior assumptions about the nature or source of the possible non-homogeneity.

Since the standard analysis of the data by fitting a kernel density estimator (see the solid curve in the right panel of Figure 1) appears to give no definite indication of multimodality, it seems, that some more sophisticated methods are needed in order to identify any foreign components of the claims. Whereas many different approaches to this difficult problem are possible, we have chosen one that shall illustrate the applicability of the EM methodology in our setting. Namely, we shall attempt to fit a log-mixture-normal distribution to the data, that is, we shail model the logarithm of the claim outpatient medical billing distribution as a mixture of several normal variables. The use of normal distributions here is mostly due to convenience of the EM implementation and in more complicated real life problems can be inappropriate. However, the principle that we shall attempt to describe here is, in general, applicable to any mixture of distributions, even including non-parametric ones. ${ }^{3}$

\footnotetext{
${ }^{3}$ The notion of fitting non-parametric distributions via likelihood methods, which at first may seem contradiction in terms, has become very popular in statistics over the last decade. This is due to intensive research into the so called empirical likelihood methods (see for instance a recent monograph by Owen 2001 and references therein). In
} 
In order to describe our method in the context of the EM algorithm we shall again relate the problem at hand to our EM methodology introduced in Section 1. In our current setting we shall consider the set of logarithms of the $\mathrm{BI}$ claim medical bills as the incomplete data $\mathcal{X}$. According to our model assumption we identify the underlying random variable $X$, of which $\mathcal{X}$ is a realization, as a mixture of several (say, $m \geq 2$ ) normal distributions ${ }^{4}$

$$
\begin{aligned}
X_{i} & \sim N\left(\mu_{j}, \sigma_{j}\right) \text { for } j=1, \ldots, m \\
X & =\sum_{j=1}^{m} Y_{j} \cdot X_{j}
\end{aligned}
$$

where $Y_{j} \in\{0,1\}$ with $P\left(Y_{j}=1\right)=\pi_{j}$ such that $\sum \pi_{j}=1$ and the joint distribution of the vector $\left(Y_{1}, \ldots, Y_{m}\right)$ is multinomial with one trial, (i.e., $\sum Y_{j}=1$ ). The right hand side of $(8)$ is sometimes known as generative representation of a mixture. Indeed, if we generate a multinomial variable $\left(Y_{1}, \ldots, Y_{m}\right)$ with probabilities of $Y_{j}=1$ equal to $\pi_{j}$, and depending on the index $j$ for which outcome is a unity, deliver $X_{j}$, then it can be shown that the density of $X$ is

$$
\sum_{j=1}^{m} \pi_{j} p\left(x \mid \Theta_{j}\right)
$$

where $p\left(\cdot \mid \Theta_{j}\right)$ is a normal density with the parameter

$$
\Theta_{j}=\left(\mu_{j}, \sigma_{j}\right) \text { for } j=1,2 \ldots, m
$$

Hence $X$ is indeed a mixture of the $X_{j}$ 's. The density given by (9) is less helpful in our approach as it doesn't explicitly involve the variables $Y_{j}$ 's. Moreover, fitting the set of parameters ${ }^{5}$

$$
\Theta=\left(\Theta_{1}, \ldots, \Theta_{m}, \pi_{1}, \ldots, \pi_{m-1}\right) .
$$

by considering log-likelihood of (9) is known to be numerically difficult as it involves evaluation of the sums under the logarithm. In contrast, the representation (8) provides for a simpler approach, which also suits better our purpose of illustrating the use of the EM methodology. In the spirit of the search for hidden exposure, we consider the (unobserved) realizations of random vector $\left(Y_{1}, \ldots, Y_{m}\right)$ in $(8)$ as the missing data $\mathcal{Y}$. Let us note that unlike in the example discussed in Section 2 here we have in some sense artificially created the set $\mathcal{Y}$. In this setting the complete set of data is now $\mathcal{Z}=(\mathcal{X}, \mathcal{Y})$ or $\mathbf{z}_{j}=\left(x_{j}, y_{j k}\right)$ for $j=1, \ldots, n$, and $k=1 \ldots, m$. Here $n=348$ is the number of obervations, $m$ is the number of components in the mixture, unspecified for now, $x_{j}$ is (logarithm of) the observed medical bill value, and $y_{j k} \in\{0,1\}$ is the auxiliary indicator variable

principle, with some modifications, the mixture approach discussed in this section and the associated EM algorithm can be applied to the empirical likelihood as well.

${ }^{4}$ Note that in our notation $\sigma$ denotes the variance, not standard deviation.

${ }^{5}$ Note that we only need to estimate $m-1$ proportions since $\sum \pi_{i}=1$. 
indicating whether or not $x_{j}$ arrives from the distribution of $X_{k}$. In this setting the complete log-likelihood function (2) takes the form

$$
l(\Theta \mid \mathcal{Z})=\sum_{j=1}^{n} \sum_{k=1}^{m} y_{j k} \log p\left(x_{j} \mid \Theta_{k}\right),
$$

and the conditional expectation ( 3 ) is given by

$$
Q\left(\Theta, \Theta^{(i-1)}\right)=\sum_{j=1}^{n} \sum_{k=1}^{m} \delta_{j k} \log p\left(x_{j} \mid \Theta_{k}\right)
$$

where

$$
\delta_{j k}=E\left(Y_{j k} \mid \Theta^{(i-1)}, \mathcal{Z}\right)=P\left(Y_{j k}=1 \mid \Theta^{(i-1)}, \mathcal{X}\right) \quad \text { for } \quad j=1 \ldots, n ; \quad k=1, \ldots, m .
$$

As we can see from the above formulae, in this particular case $Q\left(\Theta, \Theta^{(i-1)}\right)$ is obtained from the complete data likelihood by substituting for the unknown $y_{j k}$ 's their conditional expectations $\delta_{j k}$ 's calculated under the current value of the estimates of $\theta .{ }^{6}$ The quantity $\delta_{j k}$ is often referred to as the responsibility of the component $X_{k}$ for the observation $j$. This terminology reflects the fact that we may think about final $\delta_{j k}$ as the conditional (posterior) probability of the $j$-th observation arriving from the distribution of $X_{k}$.

Once we have replaced the $y_{j k}$ 's in (11) by the $\delta_{j k}$ 's, the maximization step of the EM algorithm is straightforward and applied to (12) gives the usual weighted ML estimates of the normal means, variances, and the mixing proportions (see below for the formulae). However, in order to proceed with the EM procedure we still need to construct the initial guesses for the set of parameters (10). A good way to do so (for a discussion, see, for instance, Chapter 8 of Hastie et al. 2001 or Xu and Jordan 1996) is to simply choose at random $m$ of the observed claim values as the initial estimates of the means, and set all the estimates of the variances to the overall sample variance. The mixing proportion can be set uniformly over all components. This way of initiating the parameters ensures the relative robustness of the final estimates obtained via EM against any particular initial conditions. In fact, in our BI data example we have randomly selected several initial sets of values for the means and in all case have obtained convergence to the same set of estimates. Below we present the detailed EM algorithm we have used to analyze the Massachusetts auto BI data. In order to identify the number $m$ of the mixture components in the model we have used the EM method to obtain the estimates of the complete log-likelihood function (as the final values of (12)) for $m=2,3,4$ (we have had determined earlier that for $m>4$ the BI mixture model becomes too cumbersome). The results are presented in Table 3. As can be seen from the last row of the table, $m=3$ is the number of components minimizing the negative of the estimated log-likelihood (12). Henceforth we shall, therefore, take $m=3$ for the BI mixture model.

\footnotetext{
${ }^{6}$ It may happen that some of the values $y_{j k}$ are in fact available. In such cases, we would take $\delta_{j k}=y_{j k}$.
} 
Table 3: Comparison of the mixture fit for the different values of $m$ for the BI data

\begin{tabular}{c|ccc}
\hline Parameter & $m=2$ & $m=3$ & $m=4$ \\
\hline$\mu_{1}$ & 0.071 & 0.107 & -0.01 \\
$\mu_{2}$ & 1.110 & 0.874 & 0.218 \\
$\mu_{3}$ & - & 1.248 & 0.911 \\
$\mu_{4}$ & - & - & 1.258 \\
$\sigma_{1}^{1 / 2}$ & 1.265 & 1.271 & 1.201 \\
$\sigma_{2}^{1 / 2}$ & 0.252 & 0.178 & 1.349 \\
$\sigma_{3}^{1 / 2}$ & - & 0.146 & 0.214 \\
$\sigma_{4}^{1 / 2}$ & - & - & 0.144 \\
$\pi_{1}$ & 0.470 & 0.481 & 0.250 \\
$\pi_{2}$ & 0.530 & 0.205 & 0.224 \\
$\pi_{3}$ & - & 0.314 & 0.247 \\
$\pi_{4}$ & - & - & 0.279 \\
\hline$-2 Q$ & 819.909 & 811.381 & 811.655 \\
\hline
\end{tabular}

Table 4: Selected iterations of the EM algorithm for the BI data with $m=3$.

\begin{tabular}{c|ccccccccc|c}
\hline Iteration & $\mu_{1}$ & $\mu_{2}$ & $\mu_{3}$ & $\sigma_{1}^{1 / 2}$ & $\sigma_{2}^{1 / 2}$ & $\sigma_{3}^{1 / 2}$ & $\pi_{1}$ & $\pi_{2}$ & $\pi_{3}$ & $-2 Q$ \\
\hline 1 & 0.229 & 0.785 & 0.885 & 1.172 & 0.89 & 0.843 & 0.35 & 0.329 & 0.321 & 973.115 \\
5 & -0.129 & 0.946 & 1.054 & 1.374 & 0.525 & 0.356 & 0.337 & 0.301 & 0.361 & 854.456 \\
6 & -0.131 & 0.953 & 1.083 & 1.357 & 0.499 & 0.300 & 0.349 & 0.281 & 0.370 & 839.384 \\
10 & -0.041 & 0.917 & 1.137 & 1.324 & 0.456 & 0.223 & 0.396 & 0.217 & 0.387 & 820.903 \\
20 & 0.042 & 0.875 & 1.166 & 1.302 & 0.364 & 0.207 & 0.438 & 0.177 & 0.385 & 817.363 \\
30 & 0.064 & 0.876 & 1.184 & 1.29 & 0.301 & 0.200 & 0.453 & 0.176 & 0.372 & 816.143 \\
40 & 0.074 & 0.871 & 1.204 & 1.285 & 0.259 & 0.188 & 0.460 & 0.186 & 0.354 & 814.957 \\
50 & 0.084 & 0.868 & 1.226 & 1.281 & 0.222 & 0.17 & 0.467 & 0.197 & 0.336 & 813.367 \\
60 & 0.099 & 0.871 & 1.243 & 1.275 & 0.190 & 0.153 & 0.476 & 0.204 & 0.320 & 811.838 \\
64 & 0.105 & 0.873 & 1.247 & 1.272 & 0.180 & 0.147 & 0.48 & 0.205 & 0.315 & 811.454 \\
65 & 0.107 & 0.874 & 1.248 & 1.271 & 0.178 & 0.146 & 0.481 & 0.205 & 0.314 & 811.381 \\
\hline
\end{tabular}

Algorithm 2 (The EM algorithm for fitting m-component normal mixture)

1. Define the initial estimate $\Theta^{(0)}$ of the set of parameters (10) (see discussion above).

2. The E-step: given the current value of $\Theta^{(i)}$ compute the responsibilities $\delta_{j}$ as 


$$
\delta_{j k}=\frac{\pi_{k}^{(i)} p\left(x_{j} \mid \Theta_{k}^{(i)}\right)}{\sum_{l=1}^{m} \pi_{l}^{(i)} p\left(x_{j} \mid \Theta_{l}^{(i)}\right)} \quad j=1, \ldots, n \quad \text { and } \quad k=1, \ldots, m .
$$

3. The M-step: compute the ML estimators of (12) as

$$
\pi_{k}^{(i+1)}=\frac{\sum_{j=1}^{n} \delta_{j k}}{n} \text { for } k=1, \ldots, m-1,
$$

and

$$
\begin{aligned}
\mu_{k}^{(i+1)} & =\frac{\sum_{j=1}^{n} \delta_{j k} x_{j}}{\sum_{j=1}^{n} \delta_{j k}} \\
\sigma_{k}^{(i+1)} & =\frac{\sum_{j=1}^{n} \delta_{j k}\left(x_{j}-\mu_{k}^{(i+1)}\right)^{2}}{\sum_{j=1}^{n} \delta_{j k}} \text { for } k=1, \ldots, m .
\end{aligned}
$$

4. Repeat steps 2 and 3 until the relative difference of the subsequent values of (12) is sufficiently small.

Figure 2: EM Fit. Left panel: mixture of normal distributions fitted via the $E M$ algorithm to $B I$ data. Right panel: Three normal components of the mixture. The values of all the parameters are given in the last row of Table 4.
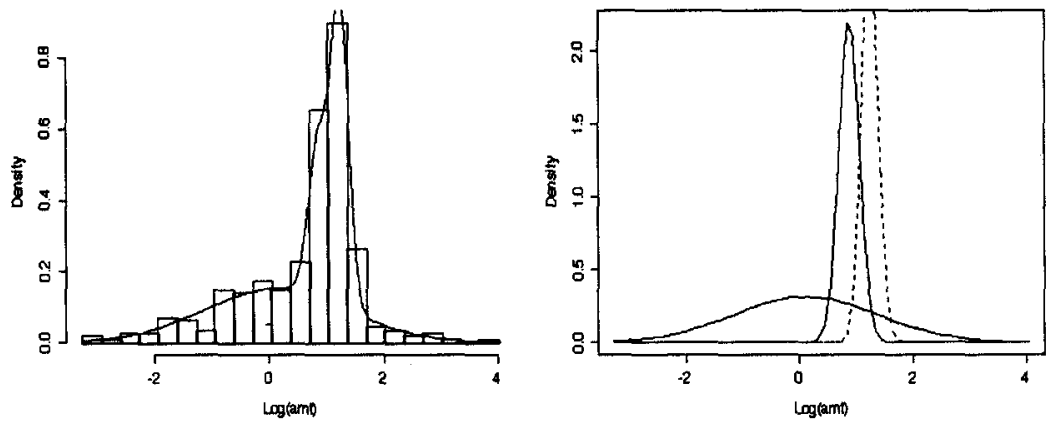

In Figures 2 and 3 we present graphically the results of the analysis of the $\mathrm{BI}$ data via the 
mixture model with $m=3$ using the EM algorithm as described above. Some selected iterations of the EM algorithm for the three component normal mixture are presented in Table 4 . In the left panel of Figure 2 we show the fit of the normal mixture fitted to the data using Algorithm 2 (with parameters values given by the last row of Table 4). As we can see the fit looks reasonable and the fitted mixture distribution looks similar to the standard density estimator (solid curve in the right panel of Figure 1). The mixture components identified by the EM method are presented in the right panel of Figure 2 and clearly indicate non-homogeneity of the data which seems to consist of two (in fact, three) different types of claims. This is, obviously, related to a high volume of claims in the interval around 1.8-4.5 thousands (corresponding to the values .6-1.5 on the log scale). This feature of the data is modeled by the two tall and thin (i.e., with small dispersion) components of the mixture (corresponding in our notation to $X_{2}$ and $X_{3}$, marked as solid and dashed curves, respectively). Let us also note the very pronounced difference (over seven-fold) in the spread between the first and the two last components.

Figure 3: Latent risk in $\mathrm{BI}$ data modeled by the $\mathrm{EM}$ algorithm with $m=3$. Left panel: set of the responsibilities $\delta_{j 3}$. Right panel: the third component of the normal mixture compared with the distribution of provider A's claims ("A" claims density estimator is a solid curve).
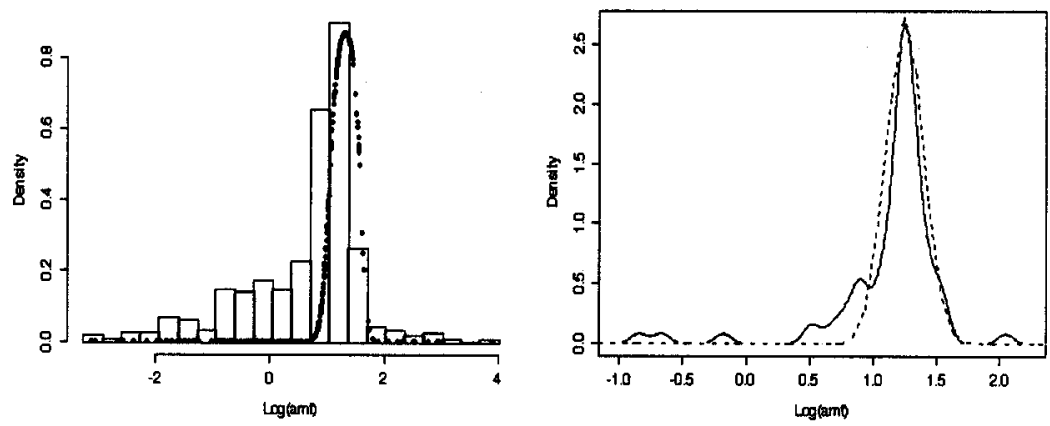

In the left panel of Figure 3 we present the set of responsibilities $\left(\delta_{j 3}\right)$ of the model (or component) $X_{3}$ as calculated by the EM algorithm superimposed on the histogram of the BI data. The numerical values of the responsibilities for each data point are also listed in the last column of the table in Appendix B. The relationship between the set of responsibilities obtained via the EM procedure and the apparent lack of homogeneity of the data, demonstrated by Figure 2, is easy to see. The high responsibilities are clustered around the claim values within two standard deviations of the estimated mean (1.25) of the tallest distribution $X_{3}$. Hence the plot of responsibilities 
superimposed on the data distribution again uncovers the non-homogeneity or the risk factor which was initially hidden. As we can see from the right panel in Figure 3 the observed nonhomogeneity may be attributed largely, as initially expected (and as the illustration intended), to the high kurtosis of "A" claims. Indeed, the superimposing of the distribution of "A" claims (solid curve) on the component $X_{3}$ (dashed curve) in the right panel of Figure 3 reveals a reasonably close match in the interval $(.8,1.7)$ or so. Outside this interval the normal approximation to the provider A's claims fails, mostly due to the fact that the normal mixture model employed is not sufficiently "fine tuned" in its tails to look for this particular type of distribution. The deficiency could be perhaps rectified in this particular case by incorporating some different (non-normal) components into the mixture model. However, our main task in this analysis was to merely uncover hidden factors (if any) and not necessarily to model them precisely, which should be done afterwards using some different, more sophisticated modeling approach depending on the type of problem at hand. See, for instance, Bilmes, (1998) who presents the extension of our Algorithm 2 to the so-called general hidden Markov model (HMM). For a full review of some possible approaches to fitting the finite mixtures models and the use of the EM methodology in this context, readers are referred to the recent monograph by McLachlan and Peel (2000) which also contains some descriptions of the currently available software for fitting a variety of non-normal mixtures via the EM method.

\subsection{The EM Algorithm Output and Fuzzy Set Membership Function}

As we have seen above, each run of the EM algorithm estimating an $m$-mixture model will produce responsibilities for each claim and for each one of the $m$ mixture distributions. As mentioned earlier, they can be interpreted as the (posterior) probability that the claim "arises" from each of the components of the mixing distributions. They also can be interpreted as the membership functions for the fuzzy sets of "arising from the $i$-th mixture component". If for any claim the responsibility (membership) of a particular model component equals one, we say that the claim arises from that model component. When the responsibility is less than one, the claim arises partially from that component, and if the responsibility equals zero, we can say the claim does not arise from that component. In that context, every claim "belongs to" each of the mixing component with measurement value equal to the responsibility. Putting the EM algorithm within the fuzzy set context provides us with the well-known tools of fuzzy arithmetic to help interpret the EM output in a way that matches real-life actuarial choices (c.f., e.g., Derrig and Ostaszewski 1999)

Another advantage of portraying the responsibility probabilities as fuzzy sets relations is that the defuzzification operator known as the $\alpha$-cut ${ }^{7}$, can be used to illustrate the type I and II errors when the $\alpha$-cut criterion is used to classify the claim as belonging to one of the mixture distributions. The $\alpha$-cut classification table is presented in Table 5 below and shows the portions of " $\mathrm{A}$ " claims contained in each $\alpha$-cut from 0.1 to 0.9 for each mixture component distribution. In particular, the $\alpha$-cut analysis confirms our previous findings that " $\mathrm{A}$ " claims belong predominantly to the third

\footnotetext{
${ }^{7}$ For $\alpha$ equal to a number between zero and one, the $\alpha$-cut of a fuzzy set consists of the (crisp) set of all elements that have a membership value greater than or equal to $\alpha$ (see, Derrig and Ostaszewski 1995).
} 
mixing distribution (i.e., distribution of $X_{3}$ ). Indeed, the $\alpha$-cut at about 0.5 provides us with a good indication that " $\mathrm{A}$ " arises from the third mixing distribution (corresponding to the value $75 \%$ in the table) but not from the first one (corresponding to $8 \%$ value only). These findings are consistent with those illustrated by Figure 3. In contrast, the second mixing distribution (distribution of $\mathrm{X}_{2}$ ) does not allow us to classify correctly " $\mathrm{A}$ " and "other" in our three-mixture model. The low proportion of "A" claims assigned to the model $X_{2}$ indicates that they are generally unlikely to arrive from $X_{2}$ which may be an indication of some further non-homogeneity among claims, even after adjusting for the type " $\mathrm{A}$ ". The $X_{2}$ component could be, therefore, the manifestation of some additional hidden factors, which again confirms the findings summarized in the previous section. ${ }^{8}$

Table 5: Fuzzy membership via responsibility probabilities

\begin{tabular}{l|ll|ll|ll}
\hline$\alpha$ & Resp. $X_{1}$ & & Resp. $X_{2}$ & & Resp. $X_{3}$ \\
& A & Other & A & Other & A & Other \\
\hline \hline 0.9 & 0.05 & 0.42 & 0.00 & 0.00 & 0.00 & 0.00 \\
0.8 & 0.05 & 0.42 & 0.00 & 0.00 & 0.54 & 0.13 \\
0.7 & 0.07 & 0.45 & 0.09 & 0.11 & 0.62 & 0.19 \\
0.6 & 0.08 & 0.46 & 0.13 & 0.20 & 0.70 & 0.22 \\
$\mathbf{0 . 5}$ & $\mathbf{0 . 0 8}$ & $\mathbf{0 . 4 8}$ & $\mathbf{0 . 1 3}$ & $\mathbf{0 . 2 4}$ & $\mathbf{0 . 7 5}$ & $\mathbf{0 . 2 4}$ \\
0.4 & 0.11 & 0.49 & 0.16 & 0.28 & 0.78 & 0.26 \\
0.3 & 0.16 & 0.54 & 0.21 & 0.30 & 0.79 & 0.30 \\
0.2 & 0.22 & 0.64 & 0.24 & 0.35 & 0.79 & 0.34 \\
0.1 & 0.95 & 1.00 & 0.33 & 0.41 & 0.82 & 0.38 \\
\hline
\end{tabular}

\subsection{Accuracy Assessment for the EM Output via Parametric Bootstrap}

In our analysis of the $\mathrm{BI}$ data conducted in the previous sections we have used the numeric values of the estimated parameters (10) and the responsibilities (13). Since these values were estimated from the data via the EM algorithm, it is important to learn about their accuracy. In general, for the set of parameters $(10)$ the usual approach to assessing accuracy based on the asymptotic normality of the maximum likelihood estimators can be applied here, as soon as we calculate the information matrix for $\Theta$. This is slightly more complicated for the set of responsibilities (13) as they are the functions of $\Theta$ and hence require the appropriate transformation of the information matrix. However, a simpler method of obtaining, for instance, confidence intervals for the set of responsibilities and the model parameters can be also used, based on the so-called parametric

\footnotetext{
${ }^{8} \mathrm{An}$ analysis of the mixture model applied only to 272 "other" claims shows that $X_{2}$ has a more pronounced representation (high $\alpha$-cut proportions) of (i) chiropractic and physical therapy treatment, (ii) special investigations and independent medical examinations, and (iii) extended treatment delays.
} 
bootstrap method outlined in Algorithm 3 below. The method can be shown to be asymptotically equivalent to the normal approximation approach and is known to be often more reliable for smaller sample sizes or for the heavily biased estimators (which will often be the case for the responsibilities (13)). The algorithm below describes how to obtain confidence intervals for the parameters given by (10) and (13) using bootstrap. For some more examples and further discussion see, for instance, McLachlan and Peel (2000) or the forthcoming paper by Rempala and Szatzschneider (2002) where also the issue of the hypothesis testing for the number of mixture components via the parametric bootstrap method is discussed.

Algorithm 3 (Bootstrap confidence intervals)

1 Using the values of the model parameters (10) obtained from the EM algorithm generate the set of pseudo-data $\mathcal{X}^{*}$ (typically of the same length as the original data $\mathcal{X}$ ).

2 With $\mathcal{X}^{*}$ at hand, use Algorithm 2 in order to obtain a set of pseudo-values $\Theta^{*}$.

3 Using the set of the original data values $\mathcal{X}$ and $\Theta^{*}$ from step 2 above, calculate the pseudoresponsibilities $\delta_{j k}^{*}$ as in Algorithm 2 step 2.

4 Repeat the steps 1-3 a large number of times, say, $B .{ }^{9}$

5 Use the empirical quantiles of the distributions of pseudo-values $\Theta^{*}$ and $\delta_{j k}^{*}$ to obtain confidence bounds for $\Theta$ and $\delta_{j k}$.

For illustration purpose we present the set of confidence intervals for the three-mixture-normal model parameters and the responsibilities (of $X_{3}$ ) obtained via the above algorithm for the $\mathrm{BI}$ data in Tables 6 and 7 below. The term "bootstrap estimate" in the tables refers to the average value of the $B$ bootstrap pseudo-values obtained in steps 2 or 3 .

\section{Summary and Conclusion}

This paper has introduced the statistical methodology for inference in the presence of missing data, known as the EM algorithm, into the actuarial settings. We have shown that this methodology is particularly appropriate for those practical situations which require consideration of the missing or incomplete data, the "lurking" variables, or the hidden factors. We believe that due to its conceptual simplicity, the EM method could become a standard tool of actuarial analysis in the future. Herein we have given only some example of its usefulness in modeling loss severity. Specifically, in modeling claim severities, the EM algorithm was used to impute missing values in a more sophisticated and statistically less biased way than simple regression methods as well as to uncover (hidden) patterns in the claim severity data. Actual auto bodily injury liability claims closed in Massachusetts in 2001 were used to illustrate a first stage screen for abusive medical providers, and

\footnotetext{
${ }^{9}$ In our setting $B$ needs to be fairly large, typically at least a thousand. For a discussion see, for instance, McLachlan and Peel (2001).
} 
Table 6: Accuracy of the parameter estimates for the $\mathrm{BI}$ data with $B=1000$

\begin{tabular}{c|ccc}
\hline Parameter & Value & $\begin{array}{c}\text { Bootstrap } \\
\text { Estimate }\end{array}$ \\
\hline \hline$\mu_{1}$ & 0.107 & 0.104 & $(-0.115,0.298)$ \\
$\mu_{2}$ & 0.874 & 0.871 & $(0.809,0.924)$ \\
$\mu_{3}$ & 1.248 & 1.249 & $(1.216,1.284)$ \\
$\sigma_{1}^{1 / 2}$ & 1.271 & 1.269 & $(1.132,1.389)$ \\
$\sigma_{2}^{1 / 2}$ & 0.178 & 0.175 & $(0.125,0.222)$ \\
$\sigma_{3}^{1 / 2}$ & 0.146 & 0.144 & $(0.117,0.174)$ \\
$\pi_{2}$ & 0.205 & 0.207 & $(0.157,0.253)$ \\
$\pi_{3}$ & 0.314 & 0.317 & $(0.268,0.375)$ \\
\hline
\end{tabular}

Table 7: Accuracy of the selected responsibilities $\delta_{j 3}$

\begin{tabular}{c|cccc}
\hline $\begin{array}{c}\text { No } \\
(j)\end{array}$ & $\begin{array}{c}\text { Log Claim } \\
\text { Value }\end{array}$ & $\begin{array}{c}\delta_{j 3} \\
\text { Value }\end{array}$ & $\begin{array}{c}\text { Bootstrap } \\
\text { Estimate }\end{array}$ & $95 \% \mathrm{Cl}$ \\
\hline \hline 100 & 0.380 & 0.000 & 0.000 & $(3.90 \mathrm{e}-12,2.04 \mathrm{e}-06)$ \\
200 & 1.031 & 0.410 & 0.396 & $(0.243,0.531)$ \\
300 & 1.353 & 0.854 & 0.863 & $(0.8020 .912)$ \\
\hline
\end{tabular}

their abusive claims, utilizing the EM algorithm. The usefulness of the EM output for classification purpose and its connections with fuzzy logic techniques were discussed. Namely, the EM algorithm output of posterior probabilities called responsibilities were reinterpreted as fuzzy set membership function in order to bring the machery of fuzzy logic to bear in the classification problem. The Monte-Carlo based method of assessing the accuracy of the model parameters fitted via the EM algorithm, known as the parametric bootstrap was also presented and the appropriate algorithm for its implementation was developed. The set of functions written in the statistical language $R$, implementing the EM algorithms discussed in the paper, have been included in Appendix $A$ to allow readers to try different actuarial situations where missing data and hidden components might be found. A large variety of actuarial and financial applications of the presented methodology are possible, including its incorporation into models of Risk Based Capital, Value at Risk, and general Dynamic Financial Analysis. We hope that this paper shall promote enough interest in the EM methodology for further exploration of those opportunities.

Acknowledgement. The authors wish to acknowledge Dr Krzysztof M. Ostaszewski, FSA for his encouragements and helpful comments at the initial stages of this project. 


\section{References}

Abrahamse, Alan F. and Stephan J. Carroll (1999). The Frequency of Excess Claims for Automobile Personal Injuries, in Automobile Insurance: Road Safety, New Drivers, Risks, Insurance Fraud and Regulation, Dionne, Georges and Claire Laberge-Nadeau, Eds., Kluwer Academic Publishers, pp. 131-150.

Bilmes, Jeff A. (1998). Gentle Tutorial of the EM Algorithm and its Application to Parameter Estimation for Gaussian Mixture and Hidden Markov Models, International Computer Science Institute. UC Berkeley.

Brockett, Patrick L., Xiaohua Xia and Richard A. Derrig (1998). Using Kohonen's Self-Organizing Feature Map to Uncover Automobile Bodily Injury Claims Fraud, Journal of Risk and Insurance, June, Volume 65 , No. 2.

Campbeil, John W., Andrew Y. Lo, and Archie Craig MacKinlay (1996). The Econometrics of Financial Markets. Princeton University Press.

Crocker, Keith J., and Sharon Tennyson (1999). Costly State Falsification or Verification? Theory and Evidence from Bodily Injury Liability Claims, in Automobile Insurance: Road Safety, New Drivers, Risks, Insurance Fraud and Regulation, Dionne, Georges and Claire Laberge-Nadeau, Eds., Kluwer Academic Publishers, pp.119-131.

Cummins, J. David, and Sharon Tennyson (1996). Controlling Automobile Insurance Costs, Journal of Economic Perspectives, Spring, Volume 6, No. 2. pp. 95-115.

D'Arcy, S. P.,Gorvett, R. W., Herbers, J. A., and Hettinger, T. E. (1997). Building a Dynamic Financial Analysis Model that Flies. Contingencies November/December, 40-45.

Dempster, Allan P., N. M. Laird and D. B. Rubin (1977). Maximum likelihood from incomplete data using EM algorithm, Journal of Royal Statistical Society Series B, Volume 39, No. 1. pp. $1-38$.

Derrig, Richard A. (2002). Insurance Fraud. Journal of Risk and Insurace, Volume 69, No. 3. pp. 271-287.

Derrig, Richard A. and K.M. Ostaszewski (1999). Fuzzy Sets Methodologies in Actuarial Science, Practical Applications of Fuzzy Technologies, Hans-Jurgen Zimmerman Eds, Kluwer Academic Publishers, Boston, (November).

Derrig, Richard A., K.M. Ostaszewski, and G.A. Rempala (2001). Applications of Resampling Methods in Actuarial Practice, Proceedings of the Casualty Actuarial Society, Volume LXXXVII, pp. 322-364.

Derrig, Richard A., and K.M. Ostaszewski (1995). Fuzzy Techniques of Pattern Recognition in Risk and Claim Classification, Journal of Risk and Insurance, Volume 62, No. 3. pp. 447-482. 
Derrig, Richard A., Herbert I. Weisberg and Xiu Chen (1994). Behavioral Factors and Lotteries Under No-Fault with a Monetary Threshold: A Study of Massachusetts Automobile Claims", Journal of Risk and Insurance, June, Volume 61, No. 2. pp. 245-275.

Embrechts, Paul, Alexander McNeil and Daniel Straumann (2000). Correlation and Dependence in Risk Management: Properties and Pitfalls, in Extremes and Integrated Risk Management, Paul Embrechts, Ed. pp 71-76. Risk Books. London.

Hastie. Trevor, Robert Tibshirani and Jerome Friedman (2001). The Elements of Statistical Learning, Springer-Verlag New York, Inc., New York.

Little, Roderick J.A., and Donald B. Rubin (1987). Statistical Analysis with Missing Data, John Wiley \& Sons, Inc., Canada.

McLachlan, Geoffrey and David Peel (2000). Finite mixture models, Wiley-Interscience, New York.

McLachlan, Geoffrey and Thriyambakam Krishnan (1997). The EM algorithm and extensions, Wiley, New York.

Major, John A., and Dan R. Riedinger (1992). EFD: A Hybrid Knowledge/Statistical -Based System for the Detection of Fraud, International Journal of Intelligent Systems. Volume 7, pp. 687-703 (reprinted Journal of Risk and Insurance, Vol 69, No.3, pp 309-324 September, 2002).

Owen, Art, B. (2001). Empirical Likelihood. Chapman and Hall. New York.

Rempala, Grzegorz A. and Konrad Szatzschneider (2002). Bootstrapping Parametric Models of Mortality. Manuscript, to appear in Scandinavian Actuarial Journal.

Wang, Shaun (1998). Aggregation of Correlated Risk Portfolios: Models and Algorithms Proceedings of the Casualty Actuarial Society, Volume LXXXIII, pp 848-939

Wu, Jeff A (1983). On the convergence properties of the EM algorithm, Annals of Statistics, Volume 11, No. 1. pp. 95-103.

$\mathrm{Xu}, \mathrm{Li}$ and Mike I Jordan (1996). On convergence properties of the EM algorithm for gaussian mixtures. Neural computation, Volume 8, pp. 129-151. 


\section{Appendix A. R Functions}

We present here the implementation of Algorithms 1 and 2 in statistical software $R$ which is a freeware version of the award winning statistical software $S+$ and is available from http://www.rproject.org. The functions below were used in the numerical examples discussed in the text.

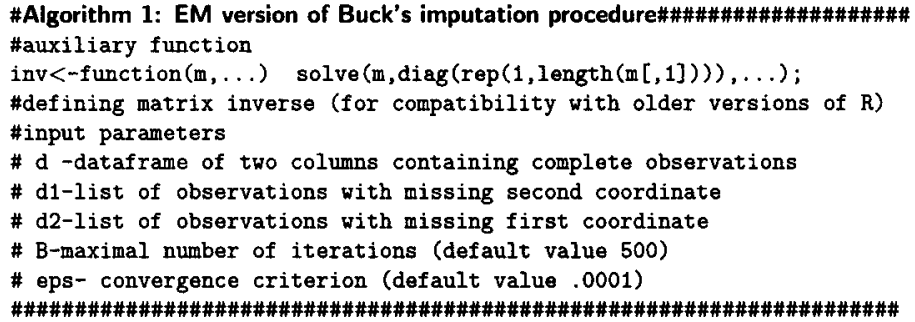


\# $\mathrm{m}$-vector of estimated means

\# R -estimated covariance matrix

\# iter -number of iterations until convergence

\# $w$-concatenated dataframe of $d, d 1, d 2$ along with imputed missing values

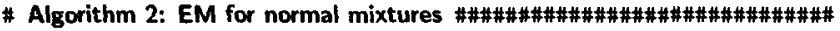

\#auxiliary function

lsum<-function (a,p.new,m, s) \{k<-length(m); ss<-0;

for $(i$ in $1: k) s s<-s s+p . n e w[[i]] * \operatorname{dnorm}(a, m[[i]], s[[i]])$;

return(ss)\}

\# facilitates calculation of L.L in the main procedure below

\# input parameters:

\# a -any list of numeric data

\# pi -initial estimate of mixing proportions (default value: uniform over three components)

\# eps -desired convergence accuracy (default value .0001)

\# B -maximal number of iterations allowed (default value 100)

\# m-initial values of means estimates (default value: random selection from a)

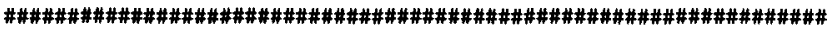

em. multnorm<-function (a, $p i=c(1 / 3,1 / 3,1 / 3)$, eps $=.0001, B=100, m=\operatorname{sort}(\operatorname{sample}(a, 3)))$

$\{\mathrm{n}<-$ length (a); k<-length (m); s<-rep(sd(a),k);

i<-1; p.new<-pi;

m0<-m;

$\log 1.01 \mathrm{~d}<-1$;

$\log 1$. new<-sum $(\log (\operatorname{lsum}(a, p$. new $, m, s)))$;

\#mainloop\#

while $($ abs $((\log 1$. new $-\log 1.01 d) / \log 1.01 d)>$ eps $i<=B)$

$\{\mathrm{g}<-\mathrm{NULL}$;

for $(t$ in $1: k) g<-r b i n d(g, p . n e w[[t]] * \operatorname{dnorm}(a, m[[t]], s[[t]]) / 1 \operatorname{sum}(a, p \cdot n \in w, m, s))$;

$m<-g \% * \%, \mathrm{a} / \mathrm{g} \% * \%$ rep $(1, \mathrm{n})$;

s<-sqrt $\left(\mathrm{g} \% * \% \mathrm{a} \sim 2 / \mathrm{g} \% * \% \operatorname{rep}(1, n)-\mathrm{m}^{\wedge} 2\right)$;

p.old<-p.new; p.new<-g\%*\% $\operatorname{rep}(1, n) / n ; i<-i+1$;

$\log 1.01 d<-\log 1$. new;

$\log 1$. new<-sum $(\log (\operatorname{lsum}(\mathrm{a}, \mathrm{p} \cdot \mathrm{new}, \mathrm{m}, \mathrm{s})))$;

\};

\#end mainloop\#

print (paste ("Theta estimates", m, s, "pi=" ,p.new, "iter=" , $i-1, "-2 L L=",-2 * \log 1$.new));

return (list $(m=m, s=s, p i=p . n e w$, ter $=i-1$, start $=m 0, \log I=-2 * \log 1$. new, resp=t $(g)$, data $a=a))\}$

\# output parameters: list of objects (m,s,pi,iter, $\log l$, resp)

\# m - vector of estimated values of means

\# s - vector of estimated values of standard deviations

\# pi -vector of estimated value of mixing proportions

\# iter- number of iterations until convergence

\# log 1 - final value of $-2 Q$

\# resp- matrix of responsibilities (columns correspond to mixture components) 


\section{Appendix B. Massachusetts Auto Insurance Bodily Injury Liability Data}

Below we present the set of Auto Insurance Data discussed in the paper. Medical bill claim amounts are given in thousands. Responsibilities $\delta_{j 3}$ are calculated according to Algorithm 2.

\begin{tabular}{|c|c|c|c|c|c|c|c|c|c|}
\hline No & $\begin{array}{l}\text { Claimed } \\
\text { Amt }\end{array}$ & $\log (A m t)$ & Provider & $\begin{array}{l}\text { Resp. } \\
\delta_{j 3}\end{array}$ & No & $\begin{array}{l}\text { Claimed } \\
\text { Amt }\end{array}$ & $\log (A m t)$ & Provider & $\begin{array}{l}\text { Resp. } \\
\delta_{j 3}\end{array}$ \\
\hline 1 & 0.045 & -3.101 & Other & 0.00 & 2 & 0.047 & -3.058 & Other & 0.00 \\
\hline 3 & 0.07 & -2.659 & Other & 0.00 & 4 & 0.075 & -2.590 & Other & 0.00 \\
\hline 5 & 0.077 & -2.564 & Other & 0.00 & 6 & 0.092 & -2.386 & Other & 0.00 \\
\hline 7 & 0.117 & -2.146 & Other & 0.00 & 8 & 0.117 & -2.146 & Other & 0.00 \\
\hline 9 & 0.14 & -1.966 & Other & 0.00 & 10 & 0.145 & -1.931 & Other & 0.00 \\
\hline 11 & 0.149 & -1.904 & Other & 0.00 & 12 & 0.165 & -1.802 & Other & 0.00 \\
\hline 13 & 0.167 & -1.790 & Other & 0.00 & 14 & 0.169 & -1.778 & Other & 0.00 \\
\hline 15 & 0.18 & -1.715 & Other & 0.00 & 16 & 0.18 & -1.715 & Other & 0.00 \\
\hline 17 & 0.199 & -1.614 & Other & 0.00 & 18 & 0.202 & -1.599 & Other & 0.00 \\
\hline 19 & 0.212 & -1.551 & Other & 0.00 & 20 & 0.225 & -1.492 & Other & 0.00 \\
\hline 21 & 0.23 & -1.470 & Other & 0.00 & 22 & 0.242 & -1.419 & Other & 0.00 \\
\hline 23 & 0.264 & -1.332 & Other & 0.00 & 24 & 0.275 & -1.291 & Other & 0.00 \\
\hline 25 & 0.285 & -1.255 & Other & 0.00 & 26 & 0.29 & -1.238 & Other & 0.00 \\
\hline 27 & 0.363 & -1.013 & Other & 0.00 & 28 & 0.384 & -0.957 & Other & 0.00 \\
\hline 29 & 0.4 & -0.916 & Other & 0.00 & 30 & 0.4 & -0.916 & Other & 0.00 \\
\hline 31 & 0.413 & -0.884 & Other & 0.00 & 32 & 0.414 & -0.882 & Other & 0.00 \\
\hline 33 & 0.416 & -0.877 & Other & 0.00 & 34 & 0.425 & -0.856 & Other & 0.00 \\
\hline 35 & 0.425 & -0.856 & Other & 0.00 & 36 & 0.43 & -0.844 & Other & 0.00 \\
\hline 37 & 0.43 & -0.844 & A & 0.00 & 38 & 0.431 & -0.842 & Other & 0.00 \\
\hline 39 & 0.45 & -0.799 & Other & 0.00 & 40 & 0.46 & -0.777 & Other & 0.00 \\
\hline 41 & 0.486 & -0.722 & Other & 0.00 & 42 & 0.5 & -0.693 & Other & 0.00 \\
\hline 43 & 0.5 & -0.693 & Other & 0.00 & 44 & 0.514 & -0.666 & A & 0.00 \\
\hline 45 & 0.531 & -0.633 & Other & 0.00 & 46 & 0.54 & -0.616 & Other & 0.00 \\
\hline 47 & 0.556 & -0.587 & Other & 0.00 & 48 & 0.564 & -0.573 & Other & 0.00 \\
\hline 49 & 0.6 & -0.511 & Other & 0.00 & 50 & 0.605 & -0.503 & Other & 0.00 \\
\hline 51 & 0.605 & -0.503 & Other & 0.00 & 52 & 0.65 & -0.431 & Other & 0.00 \\
\hline 53 & 0.66 & -0.416 & Other & 0.00 & 54 & 0.66 & -0.416 & Other & 0.00 \\
\hline 55 & 0.685 & -0.378 & Other & 0.00 & 56 & 0.69 & -0.371 & Other & 0.00 \\
\hline 57 & 0.698 & -0.360 & Other & 0.00 & 58 & 0.7 & -0.357 & Other & 0.00 \\
\hline 59 & 0.705 & -0.350 & Other & 0.00 & 60 & 0.725 & -0.322 & Other & 0.00 \\
\hline 61 & 0.74 & -0.301 & Other & 0.00 & 62 & 0.75 & -0.288 & Other & 0.00 \\
\hline 63 & 0.78 & -0.248 & Other & 0.00 & 64 & 0.785 & -0.242 & Other & 0.00 \\
\hline 65 & 0.785 & -0.242 & Other & 0.00 & 66 & 0.806 & -0.216 & Other & 0.00 \\
\hline 67 & 0.825 & -0.192 & Other & 0.00 & 68 & 0.825 & -0.192 & Other & 0.00 \\
\hline 69 & 0.83 & -0.186 & Other & 0.00 & 70 & 0.836 & -0.179 & A & 0.00 \\
\hline 71 & 0.87 & -0.139 & Other & 0.00 & 72 & 0.9 & -0.105 & Other & 0.00 \\
\hline 73 & 0.934 & -0.068 & Other & 0.00 & 74 & 0.95 & -0.051 & Other & 0.00 \\
\hline 75 & 0.954 & -0.047 & Other & 0.00 & 76 & 0.956 & -0.045 & Other & 0.00 \\
\hline 77 & 0.962 & -0.039 & Other & 0.00 & 78 & 0.97 & -0.030 & Other & 0.00 \\
\hline 79 & 0.975 & -0.025 & Other & 0.00 & 80 & 0.988 & -0.012 & Other & 0.00 \\
\hline
\end{tabular}




\begin{tabular}{|c|c|c|c|c|c|c|c|c|c|}
\hline No & $\begin{array}{l}\text { Claimed } \\
\text { Amt }\end{array}$ & $\log (A m t)$ & Provider & $\begin{array}{l}\text { Resp. } \\
\delta_{j 3}\end{array}$ & No & $\begin{array}{l}\text { Claimed } \\
\text { Amt }\end{array}$ & $\log (A m t)$ & Provider & $\begin{array}{l}\text { Resp. } \\
\delta_{j 3}\end{array}$ \\
\hline 81 & 1.015 & 0.015 & Other & 0.00 & 82 & 1.053 & 0.052 & Other & 0.00 \\
\hline 83 & 1.058 & 0.056 & Other & 0.00 & 84 & 1.08 & 0.077 & Other & 0.00 \\
\hline 85 & 1.161 & 0.149 & Other & 0.00 & 86 & 1.167 & 0.154 & Other & 0.00 \\
\hline 87 & 1.195 & 0.178 & Other & 0.00 & 88 & 1.215 & 0.195 & Other & 0.00 \\
\hline 89 & 1.242 & 0.217 & Other & 0.00 & 90 & 1.26 & 0.231 & Other & 0.00 \\
\hline 91 & 1.295 & 0.259 & Other & 0.00 & 92 & 1.31 & 0.270 & Other & 0.00 \\
\hline 93 & 1.319 & 0.277 & Other & 0.00 & 94 & 1.33 & 0.285 & Other & 0.00 \\
\hline 95 & 1.34 & 0.293 & Other & 0.00 & 96 & 1.355 & 0.304 & Other & 0.00 \\
\hline 97 & 1.39 & 0.329 & Other & 0.00 & 98 & 1.444 & 0.367 & Other & 0.00 \\
\hline 99 & 1.455 & 0.375 & Other & 0.00 & 100 & 1.463 & 0.380 & Other & 0.00 \\
\hline 101 & 1.49 & 0.399 & Other & 0.00 & 102 & 1.5 & 0.405 & Other & 0.00 \\
\hline 103 & 1.542 & 0.433 & Other & 0.00 & 104 & 1.598 & 0.469 & Other & 0.00 \\
\hline 105 & 1.616 & 0.480 & Other & 0.00 & 106 & 1.623 & 0.484 & Other & 0.00 \\
\hline 107 & 1.64 & 0.495 & Other & 0.00 & 108 & 1.645 & 0.498 & A & 0.00 \\
\hline 109 & 1.65 & 0.501 & Other & 0.00 & 110 & 1.66 & 0.507 & Other & 0.00 \\
\hline 111 & 1.68 & 0.519 & Other & 0.00 & 112 & 1.695 & 0.528 & Other & 0.00 \\
\hline 113 & 1.7 & 0.531 & A & 0.00 & 114 & 1.758 & 0.564 & Other & 0.00 \\
\hline 115 & 1.759 & 0.565 & Other & 0.00 & 116 & 1.76 & 0.565 & Other & 0.00 \\
\hline 117 & 1.896 & 0.640 & Other & 0.00 & 118 & 1.92 & 0.652 & Other & 0.00 \\
\hline 119 & 1.923 & 0.654 & Other & 0.00 & 120 & 1.941 & 0.663 & A & 0.00 \\
\hline 121 & 1.96 & 0.673 & Other & 0.00 & 122 & 1.972 & 0.679 & Other & 0.00 \\
\hline 123 & 1.99 & 0.688 & Other & 0.00 & 124 & 2.005 & 0.696 & Other & 0.00 \\
\hline 125 & 2.018 & 0.702 & Other & 0.00 & 126 & 2.02 & 0.703 & Other & 0.00 \\
\hline 127 & 2.02 & 0.703 & Other & 0.00 & 128 & 2.03 & 0.708 & Other & 0.00 \\
\hline 129 & 2.042 & 0.714 & A & 0.00 & 130 & 2.062 & 0.724 & Other & 0.00 \\
\hline 131 & 2.063 & 0.724 & Other & 0.00 & 132 & 2.08 & 0.732 & Other & 0.00 \\
\hline 133 & 2.087 & 0.736 & Other & 0.00 & 134 & 2.089 & 0.737 & Other & 0.00 \\
\hline 135 & 2.1 & 0.742 & Other & 0.00 & 136 & 2.115 & 0.749 & Other & 0.01 \\
\hline 137 & 2.12 & 0.751 & Other & 0.01 & 138 & 2.155 & 0.768 & Other & 0.01 \\
\hline 139 & 2.159 & 0.770 & Other & 0.01 & 140 & 2.161 & 0.771 & Other & 0.01 \\
\hline 141 & 2.184 & 0.781 & A & 0.01 & 142 & 2.188 & 0.783 & Other & 0.01 \\
\hline 143 & 2.191 & 0.784 & Other & 0.01 & 144 & 2.196 & 0.787 & Other & 0.01 \\
\hline 145 & 2.224 & 0.799 & Other & 0.02 & 146 & 2.237 & 0.805 & Other & 0.02 \\
\hline 147 & 2.251 & 0.811 & A & 0.02 & 148 & 2.253 & 0.812 & Other & 0.02 \\
\hline 149 & 2.288 & 0.828 & Other & 0.03 & 150 & 2.295 & 0.831 & Other & 0.03 \\
\hline 151 & 2.318 & 0.841 & Other & 0.03 & 152 & 2.325 & 0.844 & Other & 0.03 \\
\hline 153 & 2.325 & 0.844 & A & 0.03 & 154 & 2.335 & 0.848 & Other & 0.04 \\
\hline 155 & 2.341 & 0.851 & Other & 0.04 & 156 & 2.35 & 0.854 & Other & 0.04 \\
\hline 157 & 2.374 & 0.865 & Other & 0.05 & 158 & 2.39 & 0.871 & Other & 0.05 \\
\hline 159 & 2.406 & 0.878 & Other & 0.06 & 160 & 2.434 & 0.890 & Other & 0.07 \\
\hline 161 & 2.45 & 0.896 & Other & 0.08 & 162 & 2.453 & 0.897 & A & 0.08 \\
\hline 163 & 2.468 & 0.903 & Other & 0.09 & 164 & 2.468 & 0.903 & A & 0.09 \\
\hline 165 & 2.48 & 0.908 & Other & 0.10 & 166 & 2.48 & 0.908 & A & 0.10 \\
\hline 167 & 2.49 & 0.912 & A & 0.10 & 168 & 2.498 & 0.915 & Other & 0.11 \\
\hline 169 & 2.5 & 0.916 & Other & 0.11 & 170 & 2.5 & 0.916 & Other & 0.11 \\
\hline 171 & 2.5 & 0.916 & A & 0.11 & 172 & 2.51 & 0.920 & Other & 0.11 \\
\hline 173 & 2.532 & 0.929 & Other & 0.13 & 174 & 2.54 & 0.932 & Other & 0.14 \\
\hline
\end{tabular}




\begin{tabular}{|c|c|c|c|c|c|c|c|c|c|}
\hline No & $\begin{array}{l}\text { Claimed } \\
\text { Amt }\end{array}$ & $\log (A m t)$ & Provider & $\begin{array}{l}\text { Resp. } \\
\delta_{j 3}\end{array}$ & No & $\begin{array}{l}\text { Claimed } \\
\text { Amt }\end{array}$ & $\log (A m t)$ & Provider & $\begin{array}{l}\text { Resp. } \\
\delta_{j 3}\end{array}$ \\
\hline 175 & 2.543 & 0.933 & Other & 0.14 & 176 & 2.559 & 0.940 & Other & 0.15 \\
\hline 177 & 2.572 & 0.945 & Other & 0.16 & 178 & 2.593 & 0.953 & Other & 0.18 \\
\hline 179 & 2.601 & 0.956 & Other & 0.19 & 180 & 2.616 & 0.962 & Other & 0.20 \\
\hline 181 & 2.619 & 0.963 & Other & 0.20 & 182 & 2.63 & 0.967 & Other & 0.21 \\
\hline 183 & 2.635 & 0.969 & Other & 0.22 & 184 & 2.635 & 0.969 & Other & 0.22 \\
\hline 185 & 2.653 & 0.976 & Other & 0.24 & 186 & 2.655 & 0.976 & Other & 0.24 \\
\hline 187 & 2.675 & 0.984 & Other & 0.26 & 188 & 2.679 & 0.985 & Other & 0.26 \\
\hline 189 & 2.697 & 0.992 & Other & 0.28 & 190 & 2.718 & 1.000 & Other & 0.31 \\
\hline 191 & 2.73 & 1.004 & Other & 0.32 & 192 & 2.734 & 1.006 & Other & 0.32 \\
\hline 193 & 2.755 & 1.013 & Other & 0.35 & 194 & 2.758 & 1.015 & Other & 0.35 \\
\hline 195 & 2.773 & 1.020 & Other & 0.37 & 196 & 2.775 & 1.021 & Other & 0.37 \\
\hline 197 & 2.78 & 1.022 & Other & 0.38 & 198 & 2.785 & 1.024 & A & 0.38 \\
\hline 199 & 2.795 & 1.028 & Other & 0.40 & 200 & 2.805 & 1.031 & Other & 0.41 \\
\hline 201 & 2.805 & 1.031 & Other & 0.41 & 202 & 2.808 & 1.032 & A & 0.41 \\
\hline 203 & 2.88 & 1.058 & Other & 0.49 & 204 & 2.881 & 1.058 & Other & 0.50 \\
\hline 205 & 2.881 & 1.058 & A & 0.50 & 206 & 2.924 & 1.073 & A & 0.54 \\
\hline 207 & 2.93 & 1.075 & Other & 0.55 & 208 & 2.934 & 1.076 & A & 0.55 \\
\hline 209 & 2.94 & 1.078 & Other & 0.56 & 210 & 2.972 & 1.089 & Other & 0.59 \\
\hline 211 & 2.975 & 1.090 & Other & 0.59 & 212 & 3 & 1.099 & Other & 0.62 \\
\hline 213 & 3 & 1.099 & A & 0.62 & 214 & 3.025 & 1.107 & Other & 0.64 \\
\hline 215 & 3.058 & 1.118 & Other & 0.67 & 216 & 3.082 & 1.126 & A & 0.68 \\
\hline 217 & 3.085 & 1.127 & Other & 0.69 & 218 & 3.095 & 1.130 & Other & 0.69 \\
\hline 219 & 3.1 & 1.131 & Other & 0.70 & 220 & 3.102 & 1.132 & A & 0.70 \\
\hline 221 & 3.106 & 1.133 & Other & 0.70 & 222 & 3.135 & 1.143 & Other & 0.72 \\
\hline 223 & 3.17 & 1.154 & Other & 0.74 & 224 & 3.187 & 1.159 & Other & 0.75 \\
\hline 225 & 3.192 & 1.161 & A & 0.75 & 226 & 3.193 & 1.161 & Other & 0.75 \\
\hline 227 & 3.2 & 1.163 & Other & 0.76 & 228 & 3.21 & 1.166 & Other & 0.76 \\
\hline 229 & 3.23 & 1.172 & Other & 0.77 & 230 & 3.23 & 1.172 & Other & 0.77 \\
\hline 231 & 3.23 & 1.172 & A & 0.77 & 232 & 3.232 & 1.173 & Other & 0.77 \\
\hline 233 & 3.235 & 1.174 & Other & 0.78 & 234 & 3.243 & 1.176 & A & 0.78 \\
\hline 235 & 3.248 & 1.178 & A & 0.78 & 236 & 3.249 & 1.178 & Other & 0.78 \\
\hline 237 & 3.26 & 1.182 & Other & 0.79 & 238 & 3.261 & 1.182 & Other & 0.79 \\
\hline 239 & 3.272 & 1.185 & A & 0.79 & 240 & 3.29 & 1.191 & Other & 0.80 \\
\hline 241 & 3.295 & 1.192 & Other & 0.80 & 242 & 3.304 & 1.195 & Other & 0.80 \\
\hline 243 & 3.332 & 1.204 & A & 0.81 & 244 & 3.333 & 1.204 & Other & 0.81 \\
\hline 245 & 3.338 & 1.205 & Other & 0.81 & 246 & 3.34 & 1.206 & Other & 0.82 \\
\hline 247 & 3.341 & 1.206 & A & 0.82 & 248 & 3.349 & 1.209 & A & 0.82 \\
\hline 249 & 3.349 & 1.209 & A & 0.82 & 250 & 3.349 & 1.209 & A & 0.82 \\
\hline 251 & 3.353 & 1.210 & A & 0.82 & 252 & 3.36 & 1.212 & Other & 0.82 \\
\hline 253 & 3.378 & 1.217 & A & 0.83 & 254 & 3.385 & 1.219 & A & 0.83 \\
\hline 255 & 3.387 & 1.220 & A & 0.83 & 256 & 3.416 & 1.228 & Other & 0.84 \\
\hline 257 & 3.429 & 1.232 & A & 0.84 & 258 & 3.438 & 1.235 & A & 0.84 \\
\hline 259 & 3.444 & 1.237 & A & 0.84 & 260 & 3.469 & 1.244 & A & 0.85 \\
\hline 261 & 3.473 & 1.245 & A & 0.85 & 262 & 3.473 & 1.245 & A & 0.85 \\
\hline 263 & 3.475 & 1.246 & A & 0.85 & 264 & 3.477 & 1.246 & A & 0.85 \\
\hline 265 & 3.505 & 1.254 & Other & 0.85 & 266 & 3.517 & 1.258 & A & 0.85 \\
\hline 267 & 3.518 & 1.258 & Other & 0.85 & 268 & 3.527 & 1.260 & A & 0.85 \\
\hline
\end{tabular}




\begin{tabular}{|c|c|c|c|c|c|c|c|c|c|}
\hline No & $\begin{array}{l}\text { Claimed } \\
\text { Amt }\end{array}$ & $\log (A m t)$ & Provider & $\begin{array}{l}\text { Resp. } \\
\delta_{j 3}\end{array}$ & No & $\begin{array}{l}\text { Claimed } \\
\text { Amt }\end{array}$ & $\log (A m t)$ & Provider & $\begin{array}{l}\text { Resp. } \\
\delta_{j 3}\end{array}$ \\
\hline 269 & 3.535 & 1.263 & A & 0.86 & 270 & 3.547 & 1.266 & A & 0.86 \\
\hline 271 & 3.55 & 1.267 & Other & 0.86 & 272 & 3.552 & 1.268 & Other & 0.86 \\
\hline 273 & 3.567 & 1.272 & A & 0.86 & 274 & 3.57 & 1.273 & Other & 0.86 \\
\hline 275 & 3.575 & 1.274 & Other & 0.86 & 276 & 3.58 & 1.275 & Other & 0.86 \\
\hline 277 & 3.583 & 1.276 & A & 0.86 & 278 & 3.59 & 1.278 & A & 0.86 \\
\hline 279 & 3.603 & 1.282 & A & 0.86 & 280 & 3.615 & 1.285 & A & 0.86 \\
\hline 281 & 3.623 & 1.287 & $A$ & 0.86 & 282 & 3.647 & 1.294 & A & 0.86 \\
\hline 283 & 3.655 & 1.296 & Other & 0.86 & 284 & 3.655 & 1.296 & A & 0.86 \\
\hline 285 & 3.658 & 1.297 & Other & 0.87 & 286 & 3.675 & 1.302 & Other & 0.87 \\
\hline 287 & 3.675 & 1.302 & Other & 0.87 & 288 & 3.687 & 1.305 & A & 0.87 \\
\hline 289 & 3.72 & 1.314 & Other & 0.87 & 290 & 3.72 & 1.314 & Other & 0.87 \\
\hline 291 & 3.742 & 1.320 & Other & 0.87 & 292 & 3.757 & 1.324 & A & 0.87 \\
\hline 293 & 3.765 & 1.326 & Other & 0.87 & 294 & 3.8 & 1.335 & A & 0.87 \\
\hline 295 & 3.809 & 1.337 & Other & 0.86 & 296 & 3.848 & 1.348 & A & 0.86 \\
\hline 297 & 3.857 & 1.350 & A & 0.86 & 298 & 3.867 & 1.352 & Other & 0.86 \\
\hline 299 & 3.867 & 1.352 & A & 0.86 & 300 & 3.87 & 1.353 & Other & 0.86 \\
\hline 301 & 3.883 & 1.357 & Other & 0.86 & 302 & 3.89 & 1.358 & Other & 0.86 \\
\hline 303 & 3.905 & 1.362 & A & 0.86 & 304 & 3.907 & 1.363 & A & 0.86 \\
\hline 305 & 4 & 1.386 & Other & 0.85 & 306 & 4.011 & 1.389 & Other & 0.85 \\
\hline 307 & 4.039 & 1.396 & A & 0.84 & 308 & 4.065 & 1.402 & A & 0.84 \\
\hline 309 & 4.095 & 1.410 & Other & 0.83 & 310 & 4.134 & 1.419 & Other & 0.82 \\
\hline 311 & 4.147 & 1.422 & Other & 0.82 & 312 & 4.155 & 1.424 & A & 0.82 \\
\hline 313 & 4.17 & 1.428 & Other & 0.81 & 314 & 4.179 & 1.430 & A & 0.81 \\
\hline 315 & 4.2 & 1.435 & Other & 0.81 & 316 & 4.215 & 1.439 & Other & 0.80 \\
\hline 317 & 4.257 & 1.449 & A & 0.79 & 318 & 4.3 & 1.459 & Other & 0.78 \\
\hline 319 & 4.489 & 1.502 & A & 0.70 & 320 & 4.593 & 1.525 & A & 0.64 \\
\hline 321 & 4.595 & 1.525 & Other & 0.64 & 322 & 4.63 & 1.533 & A & 0.62 \\
\hline 323 & 4.653 & 1.538 & Other & 0.60 & 324 & 4.7 & 1.548 & A & 0.57 \\
\hline 325 & 4.731 & 1.554 & Other & 0.55 & 326 & 4.741 & 1.556 & A & 0.55 \\
\hline 327 & 4.75 & 1.558 & Other & 0.54 & 328 & 4.761 & 1.560 & Other & 0.53 \\
\hline 329 & 4.81 & 1.571 & Other & 0.50 & 330 & 5.072 & 1.624 & Other & 0.31 \\
\hline 331 & 5.161 & 1.641 & Other & 0.25 & 332 & 5.24 & 1.656 & Other & 0.20 \\
\hline 333 & 5.64 & 1.730 & Other & 0.06 & 334 & 5.779 & 1.754 & Other & 0.03 \\
\hline 335 & 6.166 & 1.819 & Other & 0.01 & 336 & 6.406 & 1.857 & Other & 0.00 \\
\hline 337 & 6.725 & 1.906 & Other & 0.00 & 338 & 7.717 & 2.043 & A & 0.00 \\
\hline 339 & 8 & 2.079 & Other & 0.00 & 340 & 9.5 & 2.251 & Other & 0.00 \\
\hline 341 & 10.295 & 2.332 & Other & 0.00 & 342 & 12.533 & 2.528 & Other & 0.00 \\
\hline 343 & 12.688 & 2.541 & Other & 0.00 & 344 & 16.043 & 2.775 & Other & 0.00 \\
\hline 345 & 18.847 & 2.936 & Other & 0.00 & 346 & 19.5 & 2.970 & Other & 0.00 \\
\hline 347 & 20.827 & 3.036 & Other & 0.00 & 348 & 50 & 3.912 & Other & 0.00 \\
\hline
\end{tabular}

